\title{
Clinicopathological Factors Related to the Prognosis of Metastatic Breast Cancer Patients after Development of Brain Metastasis
}

\author{
Jun Yamamuraa, ${ }^{a}$ Norikazu Masuda ${ }^{b}$ Hiroyuki Yasojima ${ }^{b}$ Makiko Mizutani ${ }^{b}$ \\ Keiko Kuriyama ${ }^{c}$ Shoji Nakamori $^{\mathrm{b}}$ Mitsugu Sekimoto $^{\mathrm{b}}$ Masayuki Mano $^{\mathrm{d}}$ Eiichi Tanaka $^{\mathrm{e}}$ \\ Masahiro Nonaka ${ }^{f}$ \\ ${ }^{a}$ Department of Surgery, Sakai City Medical Center, Osaka, Japan; \\ ${ }^{b}$ Department of Surgery and Breast Oncology, Osaka National Hospital, Osaka, Japan; \\ ${ }^{\mathrm{c}}$ Radiology, Osaka National Hospital, Osaka, Japan;

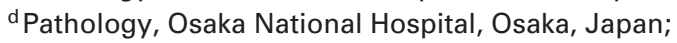 \\ e Radiation Oncology, Osaka National Hospital, Osaka, Japan; \\ ${ }^{f}$ Neurosurgery, Osaka National Hospital, Osaka, Japan
}

\section{Keywords}

Brain metastases - Breast cancer - Prognostic factor . Survival

\section{Summary}

Background: The prognosis of breast cancer patients with brain metastasis (BM) is extremely poor, and the survival after development of BM is very short. We aimed to investigate clinicopathological factors related to significant effects on the prognosis after BM development. Patients and Methods: This is a retrospective study of 75 early breast cancer patients who received the standard of care and subsequently developed BM. Results: Breast cancer subtype was one of the significant predictors for prognosis after BM diagnosis. Luminal HER2 patients had the most favorable prognosis after BM diagnosis $(p=0.011)$. Favorable performance status (PS) at BM diagnosis $(p<0.001)$ and a single metastatic brain tumor ( $p=0.032$ ) were significantly associated with good prognosis after BM diagnosis. Metastatic time courses of the patients was found not to be significantly associated with survival after BM diagnosis. Univariate and multivariate analysis indicated that luminal HER2 cancer, favorable PS at BM diagnosis, and a single metastatic brain tumor were the independent prognostic factors for survival after BM development, making a decisive influence on local or systemic control. Conclusion: Appropriate treatments for tumor subtypes and to improve the general condition of patients would result in improved outcomes for the patients with BM.

(c) 2015 S. Karger GmbH, Freiburg

\section{Introduction}

Breast cancer is a heterogeneous tumor displaying diverse characteristics including genetic, clinicopathological, and prognostic features [1-3]. The wide spectrum of these characteristics accounts for variations in response to treatments and metastatic patterns of breast cancer. The brain is one of the most common metastatic sites, with clinical and autopsy incidences of $10-16 \%$ and approximately $30 \%$ of all metastatic breast cancer [4-6]. Previous studies have identified several risk factors related to the incidence of brain metastasis (BM), such as young age, nodal status, tumor size, tumor grade, hormone receptor (HR) status - particularly negative estrogen receptor (ER), positive human epidermal growth factor receptor-2 (HER2), and triple negative (TN) phenotype [7-9]. BM exerts serious impacts on patients' daily lives, and causes severe healthcare problems. Although local treatments for intracranial lesions, including surgery, stereotactic radiosurgery, or whole brain radiation therapy, may improve quality of life for the patients, some cannot receive such local treatments due to progression of intracranial lesions. Furthermore, systemic treatments have been generally unsatisfactory, as most cytotoxic agents cannot cross the blood-brain barrier (BBB). Thus, BM results in an extremely poor prognosis for the patients.

Several clinicopathological factors have been identified to be significantly related to the prognosis after development of BM [1013]. Especially the previously published prognostic scores might be useful tools in predicting survival of breast cancer patients with $\mathrm{BM}$, which have been available for selecting appropriate treatments of BM [14-16]. They contain not only traditional prognostic fac-

\section{KARGER \\ Fax +497614520714

\section{(c) 2015 S. Karger GmbH, Freiburg}

$1661-3791 / 15 / 0106-0387 \$ 39.50 / 0$
Jun Yamamura, MD

Department of Surgery, Sakai City Medical Center

1-1-1, Ebaraji-cho, Nishi-ku

Sakai City, Osaka, 593-8304, Japan

june10th68@gmail.com 
tors on clinical features such as age, clinical stage, metastatic tumor control, performance status (PS) at the time of diagnosis of BM, and number of metastatic brain tumors, but also tumor biology including HR and HER2 status and intrinsic subtypes. However, although BM may generally occur late in the course of metastatic breast cancer, there has been little discussion about the association between time courses of metastatic cancer and survival after BM development. Identification of the time courses related to prognosis could lead to further improvement in the outcome of breast cancer patients with BM.

We analyzed the traditional clinicopathological factors as well as metastatic time courses of patients with BM, which might have an impact on survival after BM development. This retrospective study employed a database at a single institution where early breast cancer patients had received the standard of care and subsequently developed BM. Accordingly, this is very reliable for examining the association between the time courses of metastatic cancer and prognosis after BM development.

\section{Patients and Methods}

\section{Patient Characteristics}

We retrospectively reviewed the clinical records of 75 early breast cancer patients with curative resection who were histologically diagnosed as primary invasive ductal carcinoma. All patients received the standard of care and subsequently developed BM from breast cancer, as evaluated via computed tomography or magnetic resonance imaging, between February 1997 and December 2012 at Osaka National Hospital.

\section{Subtype Classification}

The presence of ER and progesterone receptors (PR) was detected by immunohistochemical (IHC) staining. Samples were considered negative

for ER and PR if $<10 \%$ of tumor cells showed expression by IHC staining. HER 2 status was analyzed by IHC staining and scored as $0,1+, 2+$, and $3+$ according to the strength of the staining. HER2 status was considered negative for staining between 0 and $1+$, and positive for $3+$. Fluorescence in situ hybridization analysis for HER2 amplification was performed for IHC $2+$ tumors. The latest gene expression studies using DNA microarrays have revealed the prognostic implications of breast cancer intrinsic subtypes [17, 18]. In this study, breast cancer subtypes were classified as follows: luminal A-like type: HR+, HER2-, and low histological grade (HG) (grade $1+2$ ); luminal B-like type: HR+, HER2-, and high HG (grade 3); luminal HER2: HR+ and HER2+; non-luminal HER2: HR- and HER2+; and TN: HR- and HER2-

\section{Survival Times and Metastatic Time Courses}

Survival time from BM diagnosis to the time of death or last follow-up was analyzed according to the clinical factors of the patients at the initial diagnosis of primary breast cancer and at the diagnosis of $\mathrm{BM}$, and time parameters in the metastatic courses, such as time from the initial diagnosis of primary breast cancer to the first distant metastasis, time from the first distant metastasis to $\mathrm{BM}$ diagnosis, and time from the initial diagnosis of primary breast cancer to $\mathrm{BM}$ diagnosis

\section{Statistical Analyses}

Survival parameters were calculated using the Kaplan-Meier method, and survival curves were compared using the log-rank test. Univariate and multivariate analyses were performed using the Cox regression model to investigate the independent effect of the clinical factors influencing survival after BM diagnosis. A 95\% confidence interval (CI) was calculated for all hazard ratios
Table 1. Baseline characteristics at the initial diagnosis of primary breast can$\operatorname{cer}(\mathrm{n}=75)$

\begin{tabular}{|c|c|c|}
\hline Characteristics & Status & \\
\hline \multirow[t]{2}{*}{ Median age (range), years } & & $50(27-79)$ \\
\hline & & $\mathrm{n}(\%)$ \\
\hline \multirow[t]{2}{*}{ Menopausal status } & pre & $32(43)$ \\
\hline & post & $43(57)$ \\
\hline TNM stage & $\mathrm{I}+\mathrm{II}$ & $1+50(68)$ \\
\hline (AJCC 7th edition) & III & $24(32)$ \\
\hline \multirow[t]{2}{*}{ Tumor size } & $\mathrm{T} \leq 2 \mathrm{~cm}$ & $11(15)$ \\
\hline & $\mathrm{T}>2 \mathrm{~cm}$ & $64(85)$ \\
\hline \multirow[t]{2}{*}{ Nodal status } & negative & $24(32)$ \\
\hline & positive & $51(68)$ \\
\hline \multirow[t]{3}{*}{ Lymphovascular invasion } & absent & $17(23)$ \\
\hline & present & $51(68)$ \\
\hline & unknown & $7(9)$ \\
\hline \multirow[t]{2}{*}{ Histological grade } & grade $1+2$ & $5+26(41)$ \\
\hline & grade 3 & $44(59)$ \\
\hline \multirow[t]{5}{*}{ Subtypes } & luminal A & $13(17)$ \\
\hline & luminal B & $11(15)$ \\
\hline & luminal HER2 & $14(19)$ \\
\hline & non-luminal HER2 & $18(24)$ \\
\hline & triple negative & $19(25)$ \\
\hline \multirow[t]{2}{*}{ Surgery } & mastectomy & $41(55)$ \\
\hline & conservation & $34(45)$ \\
\hline \multirow[t]{5}{*}{ Neo-/adjuvant treatments } & chemotherapy & $54(72)$ \\
\hline & anthracyclines & $35(47)$ \\
\hline & taxanes & $23(31)$ \\
\hline & trastuzumab & $7(9)$ \\
\hline & endocrine therapy & $32(43)$ \\
\hline
\end{tabular}

(HR) in the Cox regression analysis. We performed statistical analyses by using the Statistical Software package SPSS, v.17.0 (IBM Corp., Armonk, NY, USA)

\section{Results}

We analyzed a total of 75 early breast cancer patients who subsequently developed BM in the above-stated period. The baseline characteristics at the initial diagnosis of primary breast cancer are illustrated in table 1 .

\section{Median Survival Times and Metastatic Time Courses}

The median survival times and median times according to the metastatic time courses of the patients are shown in table 2. The median survival time after BM diagnosis to the time of death or last follow-up was 6.7 months (range 0.4-50.6 months), and the median survival time after the initial diagnosis of breast cancer was 4.6 years (range 1.3-16.5 years). The median time from the initial diagnosis of breast cancer to the first distant metastasis and to BM diagnosis were 2.0 years (range 0.2-9.1 years) and 3.8 years (range $0.2-14.1$ years), respectively. 17 patients developed BM as the first distant metastasis. Excluding these patients, the median time from the first distant metastasis to BM diagnosis was 1.4 years (range 
Table 2. Median times according to time parameters

Table 3. Median survival times (MST) after diagnosis of brain metastases (BM) according to clinical factors at the initial diagnosis of primary breast cancer

\begin{tabular}{|c|c|}
\hline Time parameters & Median (range) \\
\hline Survival time after BM diagnosis, months & $6.7(0.4-50.6)$ \\
\hline Survival time after initial diagnosis, years & $4.6(1.3-16.5)$ \\
\hline Time from initial diagnosis to 1 st distant metastasis, years & $2.0(0.2-9.1)$ \\
\hline Time from 1st distant metastasis to BM diagnosis, years ${ }^{\mathrm{a}}$ & $1.4(0.1-8.2)$ \\
\hline Time from initial diagnosis to BM diagnosis, years & $3.8(0.2-14.1)$ \\
\hline
\end{tabular}

$\mathrm{BM}=$ Brain metastasis.

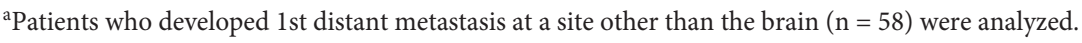

\begin{tabular}{lllll}
\hline Clinical factors & Status & $\mathrm{n}(\%)$ & MST, months & p value \\
\hline Age & $<50$ years & $34(45)$ & 9.0 & 0.460 \\
& $\geq 50$ years & $41(55)$ & 4.3 & \\
TNM stage & $\mathrm{I}+\mathrm{II}$ & $51(68)$ & 6.8 & 0.638 \\
(AJCC 7th edition) & $\mathrm{III}$ & $24(32)$ & 4.3 & \\
Tumor size & $\mathrm{T} \leq 2 \mathrm{~cm}$ & $11(15)$ & 5.0 & 0.342 \\
& T $>2 \mathrm{~cm}$ & $64(85)$ & 6.8 & \\
Nodal status & negative & $24(32)$ & 4.8 & 0.701 \\
\multirow{4}{*}{ Lymphovascular invasion } & positive & $51(68)$ & 6.8 & \\
& absent & $17(23)$ & 5.1 & 0.831 \\
& present & $51(68)$ & 6.8 & \\
Histological grade & unknown & $7(9)$ & - & 0.883 \\
& grade 1 + 2 & $31(41)$ & 4.9 & \\
Subtypes & grade 3 & $44(59)$ & 8.2 & 0.011 \\
& luminal A & $13(17)$ & 3.6 & \\
& luminal B & $11(15)$ & 6.0 & \\
& luminal HER2 & $14(19)$ & 14.5 & \\
& non-luminal HER2 & $18(24)$ & 8.8 & \\
& triple negative & $19(25)$ & 3.1 & \\
\hline
\end{tabular}

\begin{tabular}{lllrl}
\hline Clinical factors & Status & $\mathrm{n}(\%)$ & MST, months & p value \\
\hline Age & $<50$ years & $23(31)$ & 8.0 & 0.747 \\
& $\geq 50$ years & $52(69)$ & 6.5 & \\
1st metastatic site & brain & $17(23)$ & 10.5 & 0.928 \\
& others & $58(77)$ & 5.0 & \\
ECOG PS & $0-1$ & $49(65)$ & 11.5 & $<0.001$ \\
& $\geq 2$ & $26(35)$ & 1.8 & \\
Number of BM & single & $14(19)$ & 16.5 & 0.032 \\
& multiple & $61(81)$ & 5.1 & \\
Time from initial diagnosis & $<2$ years & $38(51)$ & 10.2 & 0.837 \\
$\quad$ to 1st distant metastasis & $\geq 2$ years & $37(49)$ & 5.1 & \\
Time from 1st distant metastasis & $<1.5$ years & $30(52)$ & 7.4 & 0.974 \\
$\quad$ to BM diagnosis & $\geq 1.5$ years & $28(48)$ & 3.4 & \\
Time from initial diagnosis & $<4$ years & $39(52)$ & 10.5 & 0.919 \\
$\quad$ to BM diagnosis & $\geq 4$ years & $36(48)$ & 4.4 & \\
\hline
\end{tabular}

ECOG = Eastern Cooperative Oncology Group; PS = performance status.

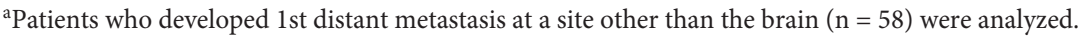

Table 4. Median survival times (MST) after diagnosis of brain metastases (BM) according to clinical factors at BM diagnosis
0.1-8.2 years). The median survival times after BM diagnosis, according to clinical factors at the initial diagnosis of breast cancer are shown in table 3. Of these factors, only breast cancer subtype was significantly related to survival after BM diagnosis. Luminal HER2 patients had the most favorable prognosis after BM diagno- sis among subtypes (HR 0.346, 95\% CI 0.165-0.724; $\mathrm{p}=0.005$ ) (fig. 1). No patient host factors at the initial breast cancer diagnosis other than tumor subtypes were significantly associated with prognosis after BM development. Table 4 shows the median survival times after BM diagnosis according to clinical factors at BM diag- 


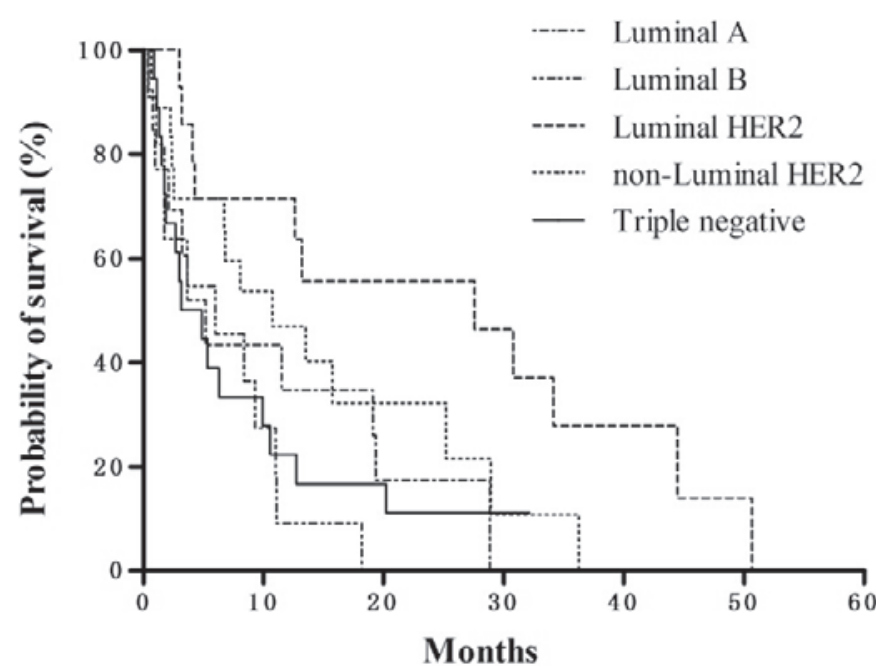

Fig. 1. Overall survival time after diagnosis of brain metastases according to breast cancer subtypes.

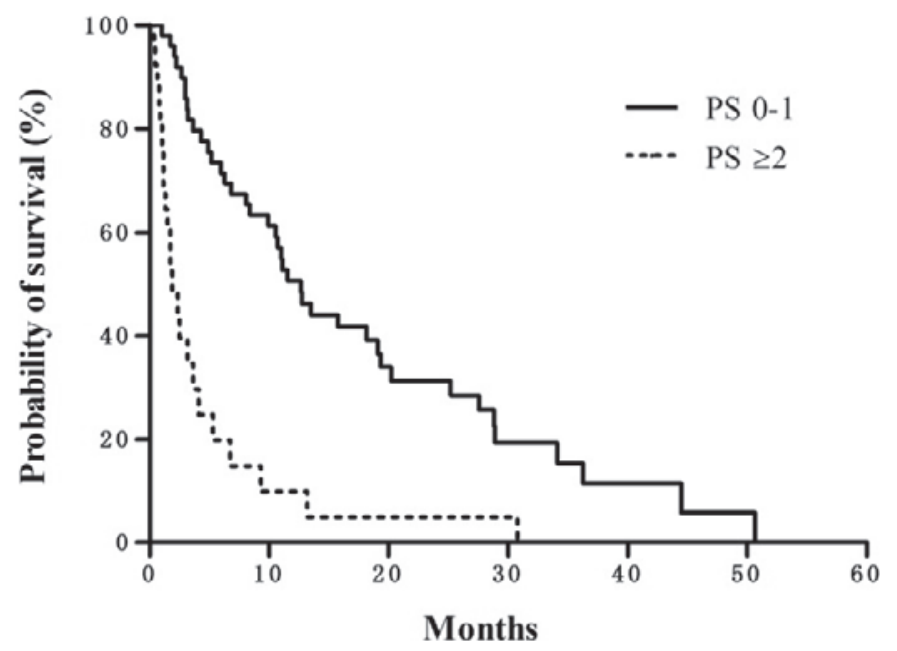

Fig. 2. Overall survival time after diagnosis of brain metastases (BM) according to performance status (PS) at the time of BM diagnosis.

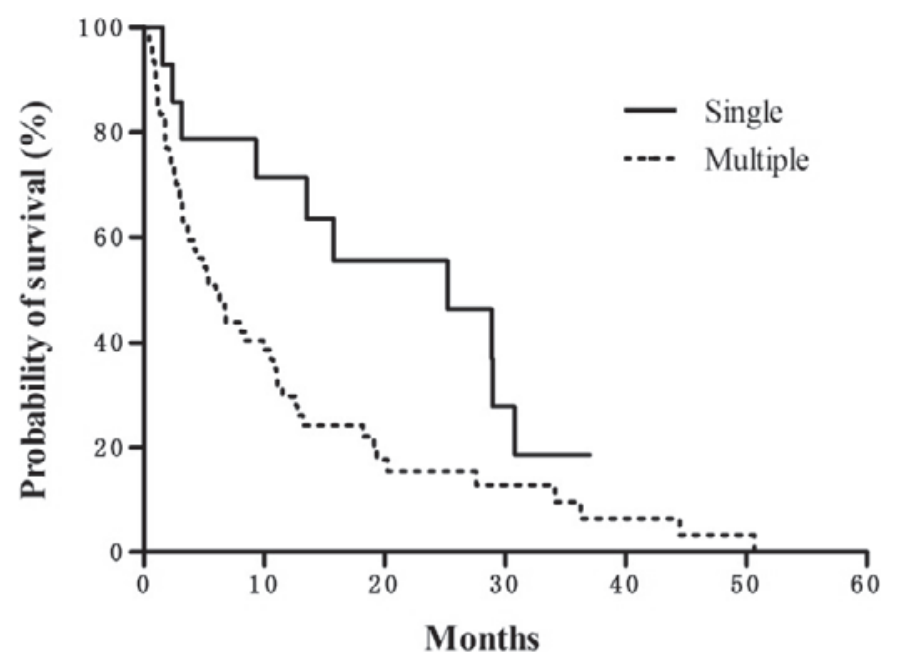

Fig. 3. Overall survival time after diagnosis of brain metastases according to the number of metastatic brain tumors. nosis and time parameters. Favorable patient PS (score 0-1) (HR 0.506, 95\% CI 0.384-0.666; $\mathrm{p}<0.001$ ) (fig. 2) and single metastatic brain tumor (HR 0.693, 95\% CI 0.491-0.978; p = 0.036) (fig. 3) were significant predictors for a better prognosis after BM development. These were decisive factors in determining not only local treatments of intracranial lesions but also systemic treatments for the patients with BM. However, age at BM diagnosis, BM as the first metastatic site, and the metastatic time courses were not significantly associated with survival after BM diagnosis.

\section{Univariate and Multivariate Analyses}

The results of the univariate and multivariate analyses are shown in table 5. Multivariate analysis revealed that luminal HER2 type (HR 0.221, 95\% CI 0.103-0.474; p < 0.001), favorable PS at BM diagnosis (HR 0.386, 95\% CI 0.281-0.531; $\mathrm{p}<0.001$ ), and single metastatic brain tumor (HR 0.601, 95\% CI 0.421-0.856; $\mathrm{p}=$ 0.005 ) were found to be independent prognostic factors for survival after BM development.

\section{Discussion}

The prognosis of metastatic breast cancer patients with BM is thought to be significantly poorer than that of those without BM [9], and the median survival time after BM development was reported to range from about 6 to 9 months [10-13]. Several studies have suggested that breast cancer subtype was one of the significant factors related to survival after BM development [10, 11]. Both HER2-positive and TN tumors have a high incidence of BM [7-9], but the former has a significantly good prognosis while the latter has a poor prognosis after BM diagnosis [9-11]. In our analysis, HER2-positive and especially luminal HER2 patients had the most favorable prognosis after BM development, relative to other subtypes. In the univariate and multivariate analysis, this type was found to be a significant independent factor for better survival after BM development. These results are in close agreement with previously published data. However, it has remained unclear why HER2-positive patients should have the most favorable outcome after BM development. HER2-positive tumors have malignant characteristics accountable for aggressive tumor growth, high risk of recurrence, and poor prognosis [19, 20], and tend to frequently develop visceral metastasis including BM [7-9]. Anti-HER2 treatments have improved survival of HER2-positive patients in the adjuvant as well as the metastatic setting $[21,22]$. Discussions have thus far been conducted regarding the relationship between antiHER2 therapy and both the incidence of BM and prognosis after BM diagnosis. Trastuzumab, a standard treatment agent for HER2positive patients, is known to have little activity for reducing intracranial lesions due to poor BBB permeability. Previous studies have indicated that treatment with trastuzumab was not associated with incidence of BM $[23,24]$, and survival after BM development was significantly longer in patients with trastuzumab than those without trastuzumab $[11,25,26]$. Trastuzumab combined with chemotherapy may favorably affect systemic metastatic lesions, 
Table 5. Univariate and multivariate analyses for prognostic factors after diagnosis of brain metastases $(\mathrm{BM})$

\begin{tabular}{llllll}
\hline & \multicolumn{2}{l}{ Univariate analysis } & & Multivariate analysis & \\
\cline { 2 - 3 } Variable & HR $(95 \% \mathrm{CI})$ & $\mathrm{p}$ value & & HR $(95 \% \mathrm{CI})$ & $\mathrm{p}$ value \\
\hline $\begin{array}{l}\text { Luminal HER2 vs. } \\
\text { others }\end{array}$ & $0.346(0.165-0.724)$ & 0.005 & & $0.221(0.103-0.474)$ & $<0.001$ \\
$\begin{array}{l}\text { ECOG PS } \\
\quad 0-1 \text { vs. } \geq 2\end{array}$ & $0.506(0.384-0.666)$ & $<0.001$ & & $0.386(0.281-0.531)$ & $<0.001$ \\
$\begin{array}{l}\text { Number of BM } \\
\quad \text { Single vs. multiple }\end{array}$ & $0.693(0.491-0.978)$ & 0.036 & & $0.601(0.421-0.856)$ & 0.005 \\
$\begin{array}{l}\text { HR = Hazard ratio; CI = confidence interval; ECOG = Eastern Cooperative Oncology Group; } \\
\text { PS = performance status. }\end{array}$ & & & & \\
\hline
\end{tabular}

probably leading to better systemic control and improved outcome of HER2-positive patients after BM development. In our study, about $80 \%$ of luminal HER2 patients continually received endocrine therapy as well as trastuzumab after BM development (data not shown), and this subtype would have more additional therapeutic options. However, it is likely that other potential factors of HER2-positive tumors may be related to favorable outcome after $\mathrm{BM}$ development, which is a topic for future discussion.

$\mathrm{BM}$ is generally considered to occur late in the course of metastatic breast cancer and was reported to occur mostly as a consequence of systemic metastasis, especially lung or liver [13, 23]. The time interval from the initial diagnosis of breast cancer to BM diagnosis was likely to be short in luminal B and TN types [10], while the time interval was showed to not be an independent prognosis factor for survival [27]. The relationship between the metastatic time courses and survival after BM diagnosis is still not well understood. Our single-institution study is very reliable for investigating the association of time parameters in the metastatic time courses with survival after BM development. However, our results showed that the time parameters had no significant correlation with survival. Other clinical host factors than the metastatic time courses might influence the prognosis of breast cancer patients with BM.

It is commonly believed that patients with BM perish as a result of not only cerebral disorder but progression of systemic disease. Thus, systemic treatments controlling the extracranial disease may be required for longer survival $[12,13]$. Patients with poor PS at $\mathrm{BM}$ diagnosis do not seem to tolerate systemic chemotherapy; accordingly, PS is a decisive factor in determining systemic treatment of the patients. Favorable PS and systematic therapy after BM development have been reported to be independent prognostic factors for overall survival after BM development [11-13]. In our study, favorable PS and a single metastatic brain tumor had a sig- nificant influence on better survival after BM diagnosis. Even when the systemic disease is under control, lack of local control of the intracranial lesion may result in poor outcomes with progressive central neurological disorders. Therefore, local treatments of intracranial lesions are essential for longer survival after BM development. Better general condition exerting a decisive influence on local or systemic control for the patients with BM was regarded as a significant factor related to favorable prognosis after BM diagnosis. Further identification of these predictors could support the selection of candidates for effective strategies, leading to better outcomes for breast cancer patients with BM.

In conclusion, luminal HER2 cancer, favorable PS at BM diagnosis, and a single metastatic brain tumor were found to be significantly related to survival after BM development and could have a decisive influence on local or systemic control. More appropriate intensive local and systemic therapies would lead to better outcomes of patients after BM development. It is hoped that such approaches may ultimately change the dismal courses of breast cancer patients with BM.

\section{Acknowledgement}

This study was supported in part by the National Cancer Center Research and Development Fund (26-A-4), and this research was partially supported by the Practical Research for Innovative Cancer Control (15ck0106049h0002) from Japan Agency for Medical Research and Development, AMED.

\section{Disclosure Statement}

The authors declare no conflicts of interest.

\section{References}

1 Perou CM, Sorlie T, Eisen MB, van de Rijn M, Jeffrey SS, Rees CA, Pollack JR, Ross DT, Johnsen H, Akslen LA, Fluge O, Pergamenschikov A, Williams C, Zhu SX, Lonning PE, Borresen-Dale AL, Brown PO, Botstein D: Molecular portraits of human breast tumours. Nature 2000;406:747-752.
Sorlie T: Molecular portraits of breast cancer: tumour subtypes as distinct disease entities. Eur J Cancer 2004; 40:2667-2675.
Van de Vijver MJ, He YD, van't Veer LJ, Dai H, Hart AA, Voskuil DW, Schreiber GJ, Peterse JL, Roberts C, Marton MJ, Parrish M, Atsma D, Witteveen A, Glas A, Delahaye L, van der Velde T, Bartelink H, Rodenhuis S, Rutgers ET, Friend SH, Bernards R: A gene-expression signature as a predictor of survival in breast cancer. New Engl J Med 2002;347:1999-2009. 
4 Lin NU, Bellon JR, Winer EP: CNS metastases in breast cancer. J Clin Oncology 2004;22:3608-3617.

5 Tsukada Y, Fouad A, Pickren JW, Lane WW: Central nervous system metastasis from breast carcinoma. Autopsy study. Cancer 1983;52:2349-2354.

6 Cho SY, Choi HY: Causes of death and metastatic patterns in patients with mammary cancer. Ten-year autopsy study. Am J Clin Pathol 1980;73:232-234.

7 Pestalozzi BC, Zahrieh D, Price KN, Holmberg SB, Lindtner J, Collins J, Crivellari D, Fey MF, Murray E, Pagani O, Simoncini E, Castiglione-Gertsch M, Gelber RD, Coates AS, Goldhirsch A: Identifying breast cancer patients at risk for central nervous system (CNS) metastases in trials of the International Breast Cancer Study Group (IBCSG). Ann Oncol 2006;17:935-944.

8 Heitz F, Rochon J, Harter P, Lueck HJ, Fisseler-Eckhoff A, Barinoff J, Traut A, Lorenz-Salehi F, du Bois A: Cerebral metastases in metastatic breast cancer: disease-specific risk factors and survival. Ann Oncol 2011;22:1571-1581.

- Nam BH, Kim SY, Han HS, Kwon Y, Lee KS, Kim TH, Ro J: Breast cancer subtypes and survival in patients with brain metastases. Breast Cancer Res 2008;10:R20.

10 Arvold ND, Oh KS, Niemierko A, Taghian AG, Lin NU, Abi-Raad RF, Sreedhara M, Harris JR, Alexander BM: Brain metastases after breast-conserving therapy and systemic therapy: incidence and characteristics by biologic subtype. Breast Cancer Res Treat 2012;136: 153-160.

11 Dawood S, Broglio K, Esteva FJ, Ibrahim NK, Kau SW, Islam R, Aldape KD, Yu TK, Hortobagyi GN, Gonzalez-Angulo AM: Defining prognosis for women with breast cancer and CNS metastases by HER2 status. Ann Oncol 2008;19:1242-1248.

12 Kim HJ, Im SA, Keam B, Kim YJ, Han SW, Kim TM, Oh DY, Kim JH, Lee SH, Chie EK, Han W, Kim DW, Kim TY, Noh DY, Heo DS, Park IA, Bang YJ, Ha SW: Clinical outcome of central nervous system metastases from breast cancer: differences in survival depending on systemic treatment. J Neurooncol 2012;106:303-313.

13 Niwinska A, Murawska M, Pogoda K: Breast cancer brain metastases: differences in survival depending on biological subtype, RPA RTOG prognostic class and systemic treatment after whole-brain radiotherapy (WBRT). Ann Oncol 2010;21:942-948.
14 Gaspar L, Scott C, Rotman M, Asbell S, Phillips T, Wasserman T, McKenna WG, Byhardt R: Recursive partitioning analysis (RPA) of prognostic factors in three Radiation Therapy Oncology Group (RTOG) brain metastases trials. Int J Radiat Oncol Biol Phys 1997;37:745-751.

15 Nieder C, Marienhagen K, Astner ST, Molls M: Prognostic scores in brain metastases from breast cancer. BMC Cancer 2009;9:105.

16 Sperduto PW, Kased N, Roberge D, Xu Z, Shanley R, Luo X, Sneed PK, Chao ST, Weil RJ, Suh J, Bhatt A, Jensen AW, Brown PD, Shih HA, Kirkpatrick J, Gaspar LE, Fiveash JB, Chiang V, Knisely JP, Sperduto CM, Lin N, Mehta M: Effect of tumor subtype on survival and the graded prognostic assessment for patients with breast cancer and brain metastases. Int J Radiat Oncol Biol Phys 2012;82:2111-2117.

17 Sorlie T, Perou CM, Tibshirani R, Aas T, Geisler S, Johnsen H, Hastie T, Eisen MB, van de Rijn M, Jeffrey SS, Thorsen T, Quist H, Matese JC, Brown PO, Botstein D, Lonning PE, Borresen-Dale AL: Gene expression patterns of breast carcinomas distinguish tumor subclasses with clinical implications. Proc Natl Acad Sci U S A 2001;98:10869-10874

18 Sorlie T, Tibshirani R, Parker J, Hastie T, Marron JS, Nobel A, Deng S, Johnsen H, Pesich R, Geisler S, Demeter J, Perou CM, Lonning PE, Brown PO, BorresenDale AL, Botstein D: Repeated observation of breast tumor subtypes in independent gene expression data sets. Proc Natl Acad Sci U S A 2003;100:8418-8423.

19 Hynes NE, Stern DF: The biology of erbB-2/neu/ HER-2 and its role in cancer. Biochim Biophys Acta 1994;1198:165-184.

20 Revillion F, Bonneterre J, Peyrat JP: ERBB2 oncogene in human breast cancer and its clinical significance. Eur J Cancer 1998;34:791-808.

21 Goldhirsch A, Gelber RD, Piccart-Gebhart MJ, de Azambuja E, Procter M, Suter TM, Jackisch C, Cameron D, Weber HA, Heinzmann D, Dal Lago L, McFadden E, Dowsett M, Untch M, Gianni L, Bell R, Kohne CH, Vindevoghel A, Andersson M, Brunt AM, Otero-Reyes D, Song S, Smith I, Leyland-Jones B, Baselga J: 2 years versus 1 year of adjuvant trastuzumab for HER2-positive breast cancer (HERA): an openlabel, randomised controlled trial. Lancet 2013;382: 1021-1028.
Swain SM, Kim SB, Cortes J, Ro J, Semiglazov V, Campone M, Ciruelos E, Ferrero JM, Schneeweiss A, Knott A, Clark E, Ross G, Benyunes MC, Baselga J: Pertuzumab, trastuzumab, and docetaxel for HER2-positive metastatic breast cancer (CLEOPATRA study): overall survival results from a randomised, double-blind, placebo-controlled, phase 3 study. Lancet Oncol 2013;14: 461-471.

23 Lai R, Dang CT, Malkin MG, Abrey LE: The risk of central nervous system metastases after trastuzumab therapy in patients with breast carcinoma. Cancer 2004;101:810-816.

24 Duchnowska R, Dziadziuszko R, Czartoryska-Arlukowicz B, Radecka B, Szostakiewicz B, Sosinska-Mielcarek K, Karpinska A, Staroslawska E, Kubiatowski T, Szczylik C: Risk factors for brain relapse in HER2-positive metastatic breast cancer patients. Breast Cancer Res Treat 2009;117:297-303.

25 Park YH, Park MJ, Ji SH, Yi SY, Lim DH, Nam DH, Lee JI, Park W, Choi DH, Huh SJ, Ahn JS, Kang WK, Park K, Im YH: Trastuzumab treatment improves brain metastasis outcomes through control and durable prolongation of systemic extracranial disease in HER2-overexpressing breast cancer patients. Br J Cancer 2009;100:894-900.

26 Park IH, Ro J, Lee KS, Nam BH, Kwon Y, Shin KH: Trastuzumab treatment beyond brain progression in HER2-positive metastatic breast cancer. Ann Oncol 2009;20:56-62.

27 Sperduto PW, Kased N, Roberge D, Chao ST, Shanley R, Luo X, Sneed PK, Suh J, Weil RJ, Jensen AW, Brown PD, Shih HA, Kirkpatrick J, Gaspar LE, Fiveash JB, Chiang V, Knisely JP, Sperduto CM, Lin N, Mehta M: The effect of tumor subtype on the time from primary diagnosis to development of brain metastases and survival in patients with breast cancer. J Neurooncol 2013; 112:467-472. 